\title{
USO DE TESTES DE VIGOR DE SEMENTES NA DETECÇÃO DE VARIABILIDADE GENÉTICA INTRACULTIVAR EM PIMENTÃO'
}

\author{
HELOISA SANTOS FERNANDES ${ }^{2}$, JORGE LUIZ NEDEL ${ }^{3}$ e JOSÉ GALLI ${ }^{3}$
}

\begin{abstract}
RESUMO - Com o objetivo de testar a hipótese de polimorfismo intracultivar para vigor em pimentão (Capsicum annuum L.), sementes de um único lote da cv. Ikeda foram submetidas a quatro testes de vigor, respectivamente: teste de frio sem solo, velocidade de germinação, envelhecimento precoce e classificação do vigor de plântula. As plântulas vigorosas e fracas, selecionadas em cada teste, junto com testemunhas (lote original, sem seleção) foram transplantadas para casa de vegetação até a produção de sementes $\mathrm{S}_{1}$. Posteriormente, comparou-se o desempenho das sementes $\mathrm{S}_{1}$ dentro do mesmo teste que possibilitara a seleção das plantas que lhes deram origem. Avaliou-se também o desempenho das sementes em relação à velocidade de emergência das plântulas em campo. Finalmente, conduziuse por mais uma geração somente as plantas vigorosas e fracas oriundas do teste de frio sem solo e testemunha - para confirmar, ou não, a existência de ganhos de seleção. Os resultados obtidos permitiram as seguintes conclusões: a) há variação quanto ao vigor individual das sementes na cultivar de pimentão utilizada; b) a variação encontrada tem, no mínimo, um componente genético passível de ser explorado, não só para aumentar a qualidade das sementes em si como para, possivelmente, melhorar o comportamento da cultivar em condições de campo; e c) o teste de frio foi o mais eficiente para detectar as diferenças comportamentais dos indivíduos dentro da população estudada.
\end{abstract}

Termos para indexação: melhoramento, germinação, polimorfismo, teste de frio.

USE OF SEED VIGOR TESTS FOR DETECTION OF INTRACULTIVAR VARIABILITY IN SWEETPEPPER

\begin{abstract}
In order to check the hypothesis of intracultivar polymorphism for vigor in sweetpepper (Capsicum annuum L.), seeds from a single lot of the cultivar Ikeda were submitted to four seed vigor tests, namely: cold test, germination rate index, accelerated aging and seedling vigor classification. Vigorous and less vigorous seedlings were separated by each test and transplanted to a greenhouse where the plants were allowed to produce seeds $\left(\mathrm{S}_{1}\right)$. As a second step, the seeds from low and high vigor seedlings were resubmitted to the test by which they were originally selected and compared to a check treatment, where selection was not made. Further, only the $\mathrm{S}_{1}$ seeds produced by the plants selected through the cold test were conducted to a second generation in order to verify possible selection gain. After data analysis it was concluded that: a) there are strong evidences indicating individual vigor variation within the seed lot of the $\mathrm{cv}$. Ikeda used; b) the evidences lead to the indication that the variation has, at least, one genetic component which may be used for breeding and seed quality improvement purposes, and c) the cold test was the most efficient for detection of individual vigor difference in the seed population.
\end{abstract}

Index terms: breeding, germination, polymorphism, cold test.

\footnotetext{
${ }^{1}$ Aceito para publicação em 20 de novembro de 1998. Extraído da Tese de Doutorado, apresentada pela primeira autora à FAEM-UFPEL.

${ }^{2}$ Enga $^{\text {a }}$ Agr $^{\mathrm{a}}$, Dra ${ }^{\mathrm{a}}$, Faculdade de Agronomia Eliseu Maciel, Universidade Federal de Pelotas (UFPEL), Caixa Postal 354, CEP 96010-970 Pelotas, RS.E-mail: heloisaf@ufpel.tche.br

${ }^{3}$ Eng. Agr., Dr., Dep. de Fitotecnia, UFPEL.
}

\section{INTRODUÇÃO}

O pimentão é conhecido mundialmente como uma das espécies que sob semeadura direta em baixas temperaturas apresenta emergência num período de tempo muito dilatado, o que leva à implantação de lavouras pouco uniformes (Gil, 1988). 
Técnicas desenvolvidas por vários pesquisadores, como: hidratação e secagem, condicionamento osmótico, incorporação de substâncias como vitaminas, aplicação de radiações, campo magnético e ultra-som, têm se constituído em tentativas para incrementar o poder germinativo e uniformizar a emergência. No entanto, por dificuldades não superadas, sua aplicação em grande escala ainda é classificada como experimental (Sampaio, 1992).

Em razão dessas observações, uma alternativa é a exploração da variabilidade genética existente, buscando uma melhor qualidade e desempenho da semente, via melhoramento das cultivares. Fato que reforça essa alternativa são as significativas diferenças entre porcentagem e tempo médio de germinação entre cultivares de pimentão, tanto em baixas como em altas temperaturas, sugerindo tratar-se de um caráter herdável (Gerson \& Honma, 1978). Revisando a literatura sobre o assunto, só foram encontradas referências sobre seleção entre linhagens e cultivares, exceção feita a Caveiro \& Gil (1991), que verificaram existência de variação na velocidade de germinação de sementes da cv. Buketen, submetidas a baixas temperaturas.

Apesar de ser considerada uma espécie preferentemente autógama, sabe-se que o pimentão pode apresentar fecundação cruzada (Diaz Rodrigues, 1990), o que poderia justificar características de heterogeneidade que resultam em desuniformidade na germinação e emergência, mesmo dentro de uma mesma cultivar. Além disso, conforme recentemente discutido por Galli (1996), é praticamente certa a existência de variação críptica em cultivares autógamas, mesmo por ocasião do seu lançamento ainda que obtida pelo método genealógico. Tal variação, entretanto, às vezes, só pode ser detectada quando, além da consciência de sua existência, forem utilizados instrumentos adequados para tanto.

O objetivo deste estudo foi verificar a ocorrência de variabilidade intracultivar na característica vigor de sementes e avaliar quais os testes de vigor seriam mais adequados para detectar diferenças entre indivíduos em uma população supostamente homogênea.

\section{MATERIAL E MÉTODOS}

O presente trabalho foi conduzido em laboratório, estufa de plástico telada e campo do Departamento de Fitotecnia da Faculdade de Agronomia "Eliseu Maciel" da Universidade Federal de Pelotas, durante o período de novembro de 1994 a julho de 1996.

Foram utilizadas sementes de pimentão de um único lote da cultivar Ikeda, safra 94, colhidas em setembro no município de Paracatu, noroeste de Minas Gerais, que após secagem e limpeza apresentaram as características relacionadas na Tabela 1.

Numa primeira etapa foram selecionadas as plântulas vigorosas e fracas, dentre 1.200 sementes, em cada um dos seguintes testes de vigor: classificação de vigor de plântulas (CVP), teste de frio sem solo (TFSS), teste de envelhecimento precoce (TEP) e teste de velocidade de germinação (TVG). Todos os testes foram realizados de acordo com as normas estabelecidas nas Regras para Análise de Sementes (Brasil, 1992).

Três repetições de 30 plântulas selecionadas em cada teste, junto com plântulas do lote original (sem seleção, testemunha), foram transplantadas para estufa de plástico telada e ali mantidas até a produção de frutos e sementes $\mathrm{S}_{1}$. Nesta fase, anotou-se altura de plantas, períodos do transplante à floração, transplante à frutificação, transplante à colheita e rendimento de frutos.

As sementes $\mathrm{S}_{1}$ foram processadas e submetidas mais uma vez, em laboratório, ao teste que lhes deu origem

TABELA 1. Caracterização da qualidade inicial do lote de sementes de pimentão (cv. Ikeda) ${ }^{\mathbf{1}}$.

\begin{tabular}{|c|c|c|c|c|c|c|c|}
\hline PMS & $\mathrm{U}$ & $\mathrm{G}$ & CVP & TFSS & TEP & TVG & TVE \\
\hline (g) & ----. & 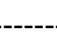 & ----- & - & --- & --------' & (índice) \\
\hline 6,59 & 8,99 & 85 & 71 & 73 & 70 & 18,06 & 8,85 \\
\hline
\end{tabular}

1 PMS: peso de mil sementes; U: umidade; G: germinação; CVP: classificação de vigor de plântulas; TFSS: teste de frio sem solo; TEP: teste de envelhecimento precoce; TVG: teste de velocidade de germinação; TVE: teste de velocidade de emergência. 
para avaliação preliminar da consistência e seletividade de cada teste. Além disso, todas as sementes $\mathrm{S}_{1}$ foram comparadas entre si em um teste de velocidade de emergência em condições de campo.

Após as avaliações, numa segunda etapa, elegeu-se somente as sementes das plantas selecionadas com o auxílio do TFSS, para análise de uma segunda geração em comparação com as das testemunhas, estudando-se o comportamento de dez plantas, em três repetições.

Nessa fase, como critérios de avaliação foram utilizados: número de dias para atingir a fase de transplante (quatro folhas), de primórdio floral, de botão floral, altura de planta, peso de matéria seca das plantas e comprimento de raiz.

Os dados obtidos foram analisados pelo programa Sistema de Análise Estatística (SANEST) (Zonta \& Machado, 1984), com as devidas transformações de dados, quando necessárias.

\section{RESULTADOS E DISCUSSÃO}

As avaliações realizadas na primeira fase revelaram que o teste de frio foi o que mostrou maior sensibilidade para detectar diferenças entre sementes com alto e baixo vigor (Tabela 2). As plantas oriundas de sementes vigorosas do teste de frio (TFv) foram mais precoces e, as de sementes menos vigo- rosas do mesmo teste (TFf), foram mais tardias do que as da testemunha e dos outros testes de vigor.

Ao submeter pela segunda vez as sementes $\left(\mathrm{S}_{1}\right)$ de plantas vigorosas e fracas ao mesmo teste que possibilitara sua seleção, observou-se novamente que o teste de frio sem solo (TFSS) foi o que apresentou maior consistência, até mesmo quanto à velocidade de emergência das plântulas em campo (IVE) (Tabela 3). Portanto, as sementes originalmente selecionadas pelo teste de frio foram as que melhor transmitiram suas características à progênie seguinte. Esses resultados confirmam os obtidos por Caveiro \& Gil (1991) em relação ao teste de frio.

A premissa básica por trás da maioria desses testes é submeter a semente a estresse, fazendo com que as fracas tenham um baixo desempenho, ao contrário das consideradas vigorosas, que deverão demonstrar um desempenho superior (Kueneman, 1982), o que sugere o teste de frio como um dos mais aplicados ao melhoramento genético de pimentão, quando o objetivo é obter ganho genético em relação a vigor de sementes.

Como o teste de frio mostrou-se o mais sensível, apenas as plantas de sementes originalmente selecionadas por esse método foram submetidas a

TABELA 2. Dados médios das observações realizadas em plantas de pimentão selecionadas com o auxílio de quatro testes de vigor, em comparação com a testemunha ${ }^{1}$.

\begin{tabular}{|c|c|c|c|c|c|}
\hline \multirow[t]{2}{*}{ Tratamento $^{2}$} & \multicolumn{3}{|c|}{ Estádio } & \multirow[t]{2}{*}{ Altura } & \multirow[t]{2}{*}{ Rendimento } \\
\hline & Floração & Frutificação & Colheita & & \\
\hline & \multicolumn{3}{|c|}{----- (dias após transplante) - } & $(\mathrm{cm})$ & (g/planta) \\
\hline Testemunha & $39,6 b c$ & $51,6 \mathrm{c}$ & 91,9abc & $55,9 \mathrm{cde}$ & $396 b$ \\
\hline TFv & $34,6 \mathrm{~d}$ & $47,3 \mathrm{~d}$ & $87,6 \mathrm{c}$ & $77,1 \mathrm{a}$ & $502 \mathrm{a}$ \\
\hline TVGv & $38,9 \mathrm{bc}$ & $50,3 \mathrm{c}$ & $90,6 a b c$ & 58,5 bcd & $383 b$ \\
\hline TEPv & $39,3 \mathrm{bc}$ & $51,9 \mathrm{bc}$ & $90,9 \mathrm{abc}$ & $62,6 b$ & $433 \mathrm{ab}$ \\
\hline TCVPv & $40,9 b c$ & $52,2 \mathrm{bc}$ & $92,6 \mathrm{ab}$ & $61,4 b c$ & $404 \mathrm{ab}$ \\
\hline TFf & $44,9 \mathrm{a}$ & $54,6 \mathrm{ab}$ & $94,6 a$ & $48,9 f$ & $350 \mathrm{~b}$ \\
\hline TVGf & $41,2 b c$ & $51,9 \mathrm{bc}$ & $91,6 a b c$ & $50,3 \mathrm{ef}$ & $360 \mathrm{~b}$ \\
\hline TEPf & $42,3 \mathrm{ab}$ & $55,9 \mathrm{a}$ & $92,6 \mathrm{ab}$ & $55,1 \mathrm{de}$ & $409 \mathrm{ab}$ \\
\hline TCVPf & $38,3 \mathrm{c}$ & $54,9 \mathrm{a}$ & $89,6 \mathrm{bc}$ & $54,7 \mathrm{def}$ & $412 \mathrm{ab}$ \\
\hline C.V. $(\%)$ & 2,241 & 1,384 & 1,344 & 7,264 & 13,639 \\
\hline
\end{tabular}

${ }^{1}$ Médias seguidas por letras distintas, na coluna, diferem significativamente pelo teste de Duncan (5\%).

2 TFv: teste de frio vigorosas; TVGv: teste de velocidade de germinação vigorosas; TEPv: teste de envelhecimento precoce vigorosas; TCVPv: teste de classificação de vigor de plântulas vigorosas; TFf: teste de frio fracas; TVGf: teste de velocidade de germinação fracas; TEPf: teste de envelhecimento precoce fracas; TCVPf: teste de classificação de vigor de plântulas fracas. 
uma segunda avaliação. Os resultados dessa avaliação se encontram na Tabela 4.

Observa-se que em todos os parâmetros avaliados, exceto o peso de matéria seca (PMS), as diferenças foram significativas entre os dois testes e a testemunha, indicando claramente o efeito da seleção divergente. As plantas oriundas de sementes selecionadas como mais vigorosas foram, respectivamente, seis e dez dias mais precoces que as não selecionadas e as selecionadas como fracas.

TABELA 3. Comparação entre todos os tratamentos no teste de velocidade de emergência das plântulas em campo, expressa pelo índice de velocidade de emergência ${ }^{1}$.

\begin{tabular}{lc}
\hline Tratamento $^{1}$ & Velocidade de emergência (índice) $^{2}$ \\
\hline Testemunha & $5,7 \mathrm{~cd}$ \\
TFv & $8,5 \mathrm{a}$ \\
TVGv & $6,6 \mathrm{bc}$ \\
TEPv & $6,9 \mathrm{~b}$ \\
TCVPv & $6,1 \mathrm{bcd}$ \\
TFf & $4,1 \mathrm{f}$ \\
TVGf & $5,2 \mathrm{de}$ \\
TEPf & $4,5 \mathrm{ef}$ \\
TCVPf & $5,9 \mathrm{bcd}$ \\
\hline C.V. $(\%)$ & 9,544 \\
\hline
\end{tabular}

1 TFv: teste de frio vigorosas; TVGv: teste de velocidade de germinação vigorosas; TEPv: teste de envelhecimento precoce vigorosas; TCVPv: teste de classificação de vigor de plântulas vigorosas; TFf: teste de frio fracas; TVGf: teste de velocidade de germinação fracas; TEPf: teste de envelhecimento precoce fracas; TCVPf: teste de classificação de vigor de plântulas fracas.

2 Médias seguidas por letras distintas, na coluna, diferem significativamente pelo teste de Duncan (5\%)
O maior peso de matéria seca observado nas plantas selecionadas como vigorosas pode estar relacionado à sua capacidade de desenvolver um sistema radicular maior e mais vigoroso. Essa característica poderia tornar a planta mais eficiente em termos de uso de água e nutrientes. Como o crescimento do sistema radicular é dependente dos fotoassimilados sintetizados na parte aérea da planta (Gardner et al., 1985), pode-se supor que essas plantas também apresentam maior eficiência fotossintética, o que contribuiria para as diferenças observadas no crescimento.

No caso específico, embora não se possa afirmar com absoluta precisão se o teste utilizado realmente aferiu diferenças de vigor herdáveis, as evidências indicam que tal ocorreu. Essa suposição se justifica pelo fato de que diferenças entre plântulas foram detectadas em todos os testes, e se tais diferenças não fossem relevantes poriam em questão a própria validade dos mesmos.

Esses resultados estão de acordo com a determinação da qualidade fisiológica da semente entre diferentes cultivares ou linhagens, em que os testes de vigor têm sido considerados os mais adequados para triagem de características direta ou indiretamente relacionadas com o vigor (Abdul-Baki \& Anderson, 1973; Gilman et al., 1973; Association of Official Seed Analysts, 1983).

A eficácia desse teste para seleção intracultivar o transforma em importante instrumento para efeitos, não só de melhoramento genético como da qualidade das sementes. Haja vista o fato de que, por tratarse de um atributo de natureza gênica complexa, sua

TABELA 4. Características das plantas de pimentão selecionadas pelo teste de frio sem solo até a fase em que iniciou a abertura das flores mais precoces ${ }^{1}$.

\begin{tabular}{|c|c|c|c|c|c|c|}
\hline Tratamento & Transplante & $\begin{array}{c}\text { Primórdio } \\
\text { floral }\end{array}$ & $\begin{array}{l}\text { Botão } \\
\text { floral }\end{array}$ & $\begin{array}{c}\text { Altura } \\
\text { de planta }\end{array}$ & Raiz & $\begin{array}{c}\text { Peso matéria } \\
\text { seca }\end{array}$ \\
\hline & \multicolumn{3}{|c|}{ 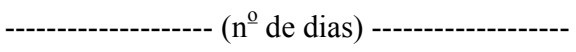 } & \multicolumn{2}{|c|}{------------ $(\mathrm{cm})$--------- } & (g/planta) \\
\hline Testemunha & 34 & $80,6 b$ & $87,6 b$ & $26,3 b$ & $30,8 b$ & $55,8 \mathrm{~b}$ \\
\hline Teste de frio vigorosas & 28 & $74,6 \mathrm{c}$ & $81,3 \mathrm{c}$ & $37,2 \mathrm{a}$ & $38,8 \mathrm{a}$ & $99,2 \mathrm{a}$ \\
\hline Teste de frio fracas & 39 & $84,6 \mathrm{a}$ & $91,6 \mathrm{a}$ & $17,3 \mathrm{c}$ & $19,9 \mathrm{c}$ & $39,2 b$ \\
\hline C.V. $(\%)$ & & 0,721 & 1,213 & 7,346 & 5,60 & 12,09 \\
\hline
\end{tabular}

${ }^{1}$ Médias seguidas por letras distintas, na coluna, diferem significativamente pelo teste de Duncan (5\%). 
introgressão, por cruzamentos, visando ao melhoramento de cultivares agronomicamente adequadas, é bastante difícil. Se utilizado na seleção de indivíduos mais vigorosos, dentro de uma cultivar já satisfatória, o melhoramento seria muito mais rápido e sem os inconvenientes da eliminação de atributos, nem sempre desejáveis, decorrentes do processo inicial de hibridação.

Tal seleção poderá, ainda, ter reflexos na obtenção de novas cultivares que, além de apresentarem estande mais uniforme, permitiriam ampliar o período de produção do pimentão, por tolerarem, durante o cultivo, temperaturas baixas que usualmente ocorrem.

No que se refere a esse tipo de avaliação (intracultivar), indicação semelhante foi sugerida por Caveiro \& Gil (1991) que selecionaram dentro da cultivar Buketen sementes com rápida e lenta germinação a $13^{\circ} \mathrm{C}$, que ao terem suas progênies testadas no campo mantiveram uma alta correlação, sugerindo que as particularidades estudadas eram herdáveis.

\section{CONCLUSÕES}

1. Há variação quanto ao vigor individual das sementes em pimentão, na cultivar Ikeda.

2. O teste de frio é o mais eficiente para detectar as diferenças comportamentais dos indivíduos dentro da população estudada.

3. A variação encontrada tem, no mínimo, um componente genético passível de ser explorado, não só para aumentar a qualidade das sementes como para, possivelmente, melhorar o comportamento da cultivar em condições de campo.

\section{REFERÊNCIAS}

ABDUL-BAKI, A.A.; ANDERSON, J.D. Vigor determination in soybean seed by multiple criteria. Crop Science, Wisconsin, v.13, n.6, p.630-633, 1973.
ASSOCIATION OF OFFICIAL SEED ANALYSTS. Seed vigor testing handbook. Lincoln, Nebraska, 1983. 93p. (Contribution, 32).

BRASIL. Ministério da Agricultura. Regras para análise de sementes. Brasília: SNAD/CLAV, 1992. 365p.

CAVEIRO, J.; GIL, R. Selección para rapida germinación a baja temperature en simiento (Capsicum annuum L.) cv. Buketen. Primeiros resultados, Actas de Horticultura, Pontevedra, v.8, p.257-262, 1991.

DIAZ RODRIGUES, G. Técnicas de la producción de semillas de plantas hortícolas. Hortofruticultura, Madrid, v.5, p.31-36, 1990.

GALLI, J. Sobre a inadequação da lei de proteção de cultivares em arroz (Oryza sativa L.). Pelotas: UFPel, 1996. 44p. Tese de Doutorado.

GARDNER, F.P.; PEARCE, R.B.; MITCHEL, R.L. Physiology of crop plants. Ames: Iowa State Univ., 1985. 327p.

GERSON, R.; HONMA, S. Emergence response of the pepper at low soil temperature. Euphytica, v.27, p.151-156, 1978.

GIL, O.R. Normas sobre extracción y conservación de semillas de pimiento como paso previo a la siembra directa. [Siembra Directa en Pimiento]. Madrid: INIA, 1988. 12p. Mesa Redonda.

GILMAN, D.F.; FEHR, W.R.; BURRIS, J.S. Temperature effects on hypocotyl elongation of soybeans. Crop Science, Wisconsin, v.13, n.2, p.246-249, 1973.

KUENEMAN, E.A. Genetic differences in soybean seed quality: screening methods for cultivar improvement. In: SINCLAIR, J.R.; JACKOBS, J.A. Soybean seed quality and stand establishment. Illinois: INTSOY, 1982. p.31-41.

SAMPAIO, T.M.G. Pré-acondicionamento osmótico y recubrimiento de semillas de pimiento (Capsicum annuum L.). Madrid: Universidad Politecnica de Madrid, 1992. 266p. Tese de Doutorado.

ZONTA, E.P.; MACHADO, A.A.P. Sistema de Análise Estatística para Microcomputadores (SANEST). Pelotas: UFPel, 1984. 127p. 